\title{
Recycling Proof Patterns in Coq: Case Studies
}

\author{
Jónathan Heras and Ekaterina Komendantskaya
}

\begin{abstract}
Development of Interactive Theorem Provers has led to the creation of big libraries and varied infrastructures for formal proofs. However, despite (or perhaps due to) their sophistication, the re-use of libraries by non-experts or across domains is a challenge. In this paper, we provide detailed case studies and evaluate the machine-learning tool ML4PG built to interactively datamine the electronic libraries of proofs, and to provide user guidance on the basis of proof patterns found in the existing libraries.
\end{abstract}

Keywords. Interactive Theorem Proving, Coq, SSReflect, Machine Learning, Clustering.

\section{Introduction}

Interactive theorem provers (ITPs) (e.g. ACL2 25], Agda [10, Coq [12, Isabelle/HOL [32], Matita 3], Mizar 17 to name a few) are a family of systems allowing the formalisation of a wide variety of domains, ranging from mathematical theories to software verification. The most recent achievements concerned formalisation and computer verification of results coming from Group Theory 15], Real Numbers 27, Discrete Mathematics [18 and Security [1]. The successful and efficient ITP programming often requires a combination of mathematical and programming intuition; see e.g. 6. There can be a rich variety of approaches to the formalisation and proof development for a given task. Thus, a programmer relies on his previous experience and ability to "creatively" adapt already used proof techniques and patterns in newly constructed proofs. The situation is similar in mathematical proofs that do not use ITPs; however, the low-level details of ITP proofs and the peculiarities of these systems make the discovery of patterns and proof techniques more difficult. This explains why a "steep learning curve" is often mentioned as one of the big obstacles to wider adoption of ITPs. In this paper, we are probing the abilities of our recent machine-learning tool ML4PG [21,22,26] to find interesting proof patterns automatically, and thus enable a more efficient use of ITPs by specialists coming from a wider range of domains.

The development of ITPs has led to the creation of big libraries and varied infrastructures for formal mathematical proofs. These frameworks usually involve thousands of definitions and theorems (for instance, there are approximately 4200 definitions and 15000 theorems in the formalisation of the Feit-Thompson theorem [15]). Parts of those libraries can often be re-applied in new domains; however, it is a challenge for expert and non-expert users alike to trace them and find re-usable concepts and proof ideas.

A different, but related, challenge is faced during the creation of these libraries. These frameworks are developed by teams (e.g. 15 people were involved in the Feit-Thompson theorem project), and the situation is similar in industry where teams use ITPs to verify the correctness of hardware and software systems. In those teams, each user has his own definitions, notation and proof-style, which

The work was supported by EPSRC grant EP/J014222/1 and EP/K031864/1. 
makes the collaborative proof development difficult. In both scenarios, it would be extremely helpful to use a tool that could detect patterns across different users, notation and libraries.

To address these challenges, we propose ML4PG - a machine-learning extension to the Proof General [4] interface for Coq [7] and its SSReflect dialect [16]. Our main goal is to prove the concept: it is possible to embed a lightweight statistical machine-learning tool into an ITP proof interface, and use it interactively to find non-trivial patterns in existing proofs and thus aid new proof developments.

The ML4PG package for Proof General features the following main functions:

- The user works within the interactive environment of Coq/SSReflect, and has an option to call ML4PG from the Proof General interface whenever he needs to see similar proof patterns.

- Based on the user's choice, ML4PG compiles the chosen libraries, and extracts significant proof features from the existing lemmas and proofs;

- ML4PG connects to machine-learning tools, and runs a number of experiments on clustering the data for each user query. Based on the results, it chooses the most reliable patterns; thus relieving the Coq programmer of the laborious step of post-processing the statistical results.

- If the user chooses to see only patterns related to his current proof goal, ML4PG would further filter the results and show the families of related proofs to the user.

- There are two ways in which we can read the results. The related theorems/lemmas are by default displayed in a separate window. Additionally, ML4PG can display the proof pattern in the form of an automaton, showing the correlating features that made the pattern.

Section 2 gives an overview of ML4PG features, details of its implementation are given in [26]. ML4PG's features have been substantially extended since [26], a detailed description of the new features is given in $[22$. In this paper, we do not focus on the ML4PG implementation per se, although we use it for all the examples and experiments shown in this paper. Our main goal here is to show how useful the automated proof pattern detection can be in different domains.

To illustrate this, we devise three experiments ("user scenarios") to test ML4PG. Each example is designed to demonstrate a different aspect of proof-pattern recognition. To demonstrate ML4PG's ability to adapt to different domains, we deliberately illustrate each user scenario by using libraries coming from different subject areas, ranging from basic mathematical infrastructures to software verification.

User Scenario 1 illustrates how to use ML4PG for detecting proof patterns prior to the start of a new proof development. To achieve this, Section 3 analyses fundamental libraries that are common in most developments using the SSReflect library [16]. The SSReflect library was developed as the infrastructure for the formalisation of the Four Colour Theorem [14 and has played a key role in the formal proof of the Feit-Thompson theorem [15]. Up to version 1.4, the SSReflect library was distributed together with the MathComp library (that contains the theories about the development of the proof of the Feit-Thompson theorem); from version 1.5, the SSReflect library can be downloaded independently from the MathComp library.

In this first scenario, we use pattern recognition with the aim of spotting common proof patterns across fundamental libraries (1404 theorems). The benefits of using ML4PG in this context is that it can be used to speed up the beginning of a proof development, making it easier to recycle patterns already available in the libraries.

User Scenario 2 considers the problem of proof-pattern discovery in a different light. In User Scenario 1, there was always an interesting underlying proof pattern hidden in the big proof libraries, "waiting" to be discovered. What if, despite the user's hope that one library may contain similar proof strategies to another, the actual proofs are in fact too different to be recycled? Section 4 studies the results that ML4PG obtains working with two different Coq libraries formalising results from game theory 34. 35. One might hope that they contain similar proof patterns, since they formalise the same subject domain; but in fact, ML4PG shows that the actual proof strategies used in [34] and [35] are completely different. This "negative" output given by ML4PG may in reality save the user's time inspecting these libraries manually. 
User Scenario 3 considers the situation when a team of several people develops a set of different modules within one bigger (industrial-scale) verification effort, see Section 5 . For this purpose, we translate the proofs of correctness of the Java Virtual Machine (JVM) given in 23] into Coq. The industrial scenario of interactive theorem proving may differ significantly from the scenarios above. Namely, industrial verification tasks often feature a bigger number of routine cases and similar lemmas; and also such tasks are distributed across a team of developers. Here, the inefficiency of automated proving often arises when programmers use different notation to accomplish very similar tasks, and thus a lot of work gets duplicated, see also 11]. We tested ML4PG in exactly such a scenario: we assumed that a programming team has collectively developed proofs of a) soundness of specification, and b) correctness of implementation of Java byte code for a dozen of programs computing multiplication, powers, exponentiation, and other arithmetic functions. We assumed that there is a relative novice in the team, trying to "learn" from the previous team efforts, in order to repeat their proof steps for a new Java function (factorial in our setting). He calls ML4PG, which discovers common patterns among these proofs and relevant lemmas (around 150 training examples in total). The suggested clusters indeed helped to advance the proofs of properties a) and b) for the Java byte code of the factorial function.

This is the first detailed evaluation of ML4PG, note that 26 focused mainly on the user interface and contained very simple examples. The case studies presented here convince us that when ML4PG statistically discovers proof clusters, it does actually find meaningful, non-trivial and interesting patterns in proofs across different libraries, theories and users. This kind of proof analysis can facilitate the use of Coq to domain expert users, see Section 6. ML4PG works on the background of Proof General, and if called, provides clustering results almost instantly; thus, can be used interactively, as a handy tool on request. Finally, it may be used for educational purposes, as automated proof-pattern recognition may help to smooth the learning curve, see User Scenario 3.

ML4PG and all examples presented in this paper are available in 21.

Related Work. ML4PG's originality is two-fold, as it can be compared to alternative methods of using machine-learning in automated theorem proving, as well as to Coq/SSReflect tools allowing interactive pattern-search.

Related work on using machine-learning in ITPs concerned hints in lemma generation for Isabelle/HOL [24], proof strategy discovery in Isabelle/HOL [5], speed up in proof automation in Mizar 28] and statistical tactic analysis in Isabelle 13]. Comparing to these tools, we use unsupervised, rather than supervised, learning; and we do not use sparse machine-learning methods. (See also 26] for a detailed comparison of different machine learning tools applied in various theorem provers.) We do not have a quantitative target when it comes to improving interactive proof building experience: neither speed up in automated proof search nor the number of automatically proven theorems are the main criteria of success. Instead, the user experience is the main parameter we target. We generally follow the "qualitative" intuition that ML4PG, being an interactive hint generator, must provide interesting and non-trivial hints on user's demands, and should be flexible and fast enough to do so in real time, at any stage of the proof, and relative to any chosen proof library.

Comparing to some of the above approaches, ML4PG does not only analyse the lemma statements, but also involves user tactics and user-defined proof-steps into the statistical proof-pattern recognition process. This feature also makes ML4PG sensitive (or adaptable) to proof styles innate to a particular user, research community, or subject area (cf. Sections 3 5). In illustration of this point, User Scenario 3 and Section 5 consider cases when different lemma statements have similar proofs; User Scenario 2 and Section 4 discuss cases when similar lemma statements require a completely different proof strategy.

Comparing to symbolic methods of proof pattern search in Coq/SSReflect, e.g. SearchPattern, Search, SearchAbout, SearchRewrite [12,16 and Whelp [2], ML4PG's originality is in introducing statistical pattern-recognition into the rich family of existing searching mechanisms in Coq/SSReflect. Unlike symbolic pattern-search, ML4PG can discover "unexpected" proof-patterns that go beyond the patterns the user would try as a searching template when using symbolic pattern-search facilities. 
Whereas the existing Coq searching tools try to match the user-provided template with other lemma/theorem statements, ML4PG takes into consideration the proof statistics in conjunction with the lemma shapes. These two features - pattern-search without a pre-defined template and the attention to the various proof parameters - allow to achieve results often orthogonal to the symbolic pattern search.

\section{Overview of ML4PG}

In this section, we present the main functionality that ML4PG offers to the user. ML4PG works with Coq and its SSReflect dialect, and it does not assume any machine-learning knowledge from the user. The guidance it provides may come in different forms. The user may prefer the statistical hint to be related to the current proof-step (cf. User Scenario 3), or give information about proof-patterns arising in a library irrespective of the current proof-step (cf. User Scenarios 1 and 2). The user may choose to data-mine only the current library, or a number of proof-libraries coming from different domains or different users. Finally, the user may wish to experiment with proof clusters of different sizes or with different machine-learning algorithms, see Table 1. These choices are accommodated within ML4PG, see 26] for a detailed description of the user interface.

ML4PG functionality is achieved in the following way.

F.1. It works on the background of Proof General extracting some low-level features from proofs in Coq/SSReflect.

F.2. It automatically sends the gathered statistics to a chosen machine-learning interface and triggers execution of a clustering algorithm according to the choice of the user.

F.3. It does some post-processing of the results given by the machine-learning tool, and displays families of related proofs to the user; on request, it shows an automaton explaining which proof features determined the pattern.

Stage F.1 is devoted to collecting statistics from proofs. The discovery of statistically significant features in data is a research area of its own in machine-learning, known as feature extraction, see [8. Statistical machine-learning algorithms classify given examples seen as points in an $m$-dimensional space, where $m$ is the maximum number of features each example may be characterised by. Irrespective of the particular feature extraction algorithm used, most pattern recognition tools [8] will require that the number of selected features is limited and fixed - the exception to this is a special class of methods called "sparse" methods [9].

ML4PG has its own feature extraction method that collects statistics from the interaction between the user and the prover. The feature extraction is done at the time of the interactive proof construction in the current library or during the Coq compilation for an external library. The feature extraction method captures information from proofs based on the correlation of a few chosen parameters within five proof steps. For each proof step, the parameters are:

1-2 the names and the number of tactics used in one command line,

3 types of the tactic arguments;

4 relation of the tactic arguments to the (inductive) hypotheses or library lemmas,

5-7 three top symbols in the term-tree of the current subgoal, and

8 the number of subgoals each tactic command-line generates.

When the correlation of these few parameters is taken within a few proof-steps, the arising statistic reveals patterns that can tell a lot about the "meta" proof strategy expressed by the tactics and subgoals. Coq proofs have different lengths and one small proof may potentially resemble a fragment of a bigger proof; also, various small "patches" of different big proofs may resemble. We address this issue by implementing an automatic split of each proof into proof-patches, thus allowing ML4PG to analyse a proof by the properties of the patches that constitute the proof. The details and discussion of this feature-extraction method can be found in 22 26]. We will not focus on the technical details of the ML4PG feature extraction here, but rather concentrate in the coming sections on proving 
the point that these simple statistical parameters (40 for one proof patch of five possibly composite proof steps) can indeed capture some essential proof-strategies, interesting and helpful enough from the user's perspective.

Once all features are extracted, ML4PG is ready to communicate with machine learning interfaces (Stage F.2). ML4PG is built to be modular - that is, the feature extraction is first completed within the Proof General environment, where the data is gathered in the format of hash tables, and then these tables are converted to the format of the chosen machine-learning tool. In [26], we connected ML4PG to several machine-learning algorithms available in Matlab 31] and Weka 19]; the results that we obtained with both systems were similar and Weka has the advantage of being an open-source software; hence, we use only Weka throughout this paper, but see 26] for a discussion of Matlab facilities.

ML4PG offers a choice of pattern-recognition algorithms. ML4PG is connected only to clustering algorithms 8] - a family of unsupervised learning methods. Unsupervised learning is chosen when no user guidance or class tags are given to the algorithm in advance: in our case, we do not expect the user to "tag" the library proofs in any way. Clustering techniques divide data into $n$ groups of similar objects (called clusters), where the value of $n$ is provided by the user. There are several clustering algorithms available in Weka (K-means, FarthestFirst and Expectation Maximisation, in short E.M.) and the user can select the algorithm using the ML4PG menu included in the Proof General interface. We show the effect of changing clustering algorithms in Table 1.

As will be illustrated in the later sections, various numbers of clusters can be useful: this may depend on the size of the Coq library, and on existing similarities between the proofs. ML4PG has its own algorithm that determines the optimal number of clusters interactively, and based on the library size. As a result, the user does not provide the value of $n$ directly, but just decides on granularity in the ML4PG menu, by selecting a value between 1 and 5, where 1 stands for a low granularity (producing fewer large clusters) and 5 stands for a high granularity (producing many smaller clusters). Given a granularity value $g$, the number of clusters $n$ is given by the formula

$$
n=\left\lfloor\frac{\text { objects to cluster }}{10-g}\right\rfloor .
$$

It is worth mentioning that it is the nature of statistical methods to produce results with some probability, and not being able to provide guarantees that a certain cluster will be found for a certain library. However, ML4PG ensures quality of the output in several different ways (Stage F.3). Results of one run of a clustering algorithm may differ from another, even on the same data set. This is due to the fact that clustering algorithms randomly choose examples to start from, and form clusters relative to those examples. However, it may happen that certain clusters are found repeatedly - and frequently - in different runs; then, we can use these frequencies to determine the reliable clusters. In particular, ML4PG output shows a digest of clustering results coming from 200 runs of the clustering algorithm. The 200 runs were experimentally found to be optimal for noticing important statistics in ML4PG setting. Only clusters that appear frequently enough are displayed to the user. There is a way to manipulate the frequency threshold within ML4PG, see [26]. Another measure is a proximity value assigned by clustering algorithms to every term in a cluster - the value ranges from 0 to 1 , and indicates the certainty of the given example belonging to the cluster. This proximity value is also taken into account by ML4PG before the results are shown. If a lemma is contained in several clusters, proximity and frequency values are used to determine one "most reliable" cluster to display.

ML4PG output has been recently enhanced with two new features 22. In addition to the families of similar proofs, ML4PG shows the key features whose correlation determined the cluster. Additionally, ML4PG uses this information to generate an automaton-shape representation for discovered proof-patterns and the correlated features, see Figures 1, 2, 4,6 .

We refer to the ML4PG user manual [21] for a more detailed description of how to use the tool. 


\section{User Scenario 1. Detecting Patterns in Early-Stages of the Development}

Users of ITPs usually start their developments loading some libraries. Those libraries contain definitions, lemmas and theorems that will be used as background theory during the proof process. Some of those libraries are specific for concrete theories, but others are common for almost every development. The common libraries contain strategies and definitions that can be extrapolated to other contexts; however, detecting lemmas that follow a concrete proof-strategy can be a challenge. In this first scenario, we study the patterns that appear in the SSReflect library 16].

The second purpose of this section is to set terminology and the general style of statistical proof-pattern analysis we will use throughout other sections.

The SSReflect library extends the Coq proof language and consists of 7 files containing basic theories about: natural numbers, lists, booleans, functions, finite types, choice types and types with a decidable equality. The library contains a total of 1404 theorems; therefore, a manual inspection of these theorems to detect patterns is unfeasible. In our first scenario, we test how ML4PG can be used to detect patterns in the SSReflect library.

We analyse clusters that are produced in the SSReflect library using the K-means algorithm and the value 5 as granularity parameter, these options produce the best results in our experiments. ML4PG discovers 280 clusters using those parameters. In $45 \%$ of those clusters (126 clusters), all the lemmas belong to the same library. We call a cluster homogeneous if it contains lemmas and theorems from one library, and heterogeneous if it contain objects from different libraries.

The mean size of the homogeneous clusters are 4 elements, and the similarities of the lemmas of a cluster can be easily spotted in most of the cases. From the 126 clusters, we can distinguish the following classification of clusters (see also 21 for a supporting extended note about this experiment).

- $36 \%$ of the clusters consist of lemmas about related functions.

Example 3.1. Examples of this kind of clusters are the ones including lemmas about: take and drop (take takes the first $n$ elements of a list and drop removes the first $n$ elements of the list):

Lemma map_take $s: \operatorname{map}($ take no $s$ ) = take no (map $s$ ).

Lemma map_drop $\mathrm{s}: \operatorname{map}(\operatorname{drop} \mathrm{n} 0 \mathrm{~s})=\operatorname{drop}$ n0 (map $\mathrm{s})$.

- $20 \%$ of clusters contain lemmas that follow the same proof structure and that share some common auxiliary results.

Example 3.2. Examples of this kind of cluster appears in several libraries, for instance in the seq library:

Lemma has_map a $s:$ has a (map $s$ ) = has (preim $f$ a) $s$.

Proof. by elim: $s=>/ /=x$ s $\rightarrow$. Qed.

Lemma all_map a $s:$ all a $(\operatorname{map} s)=\operatorname{all}($ preim $f$ a) $\mathbf{s}$.

Proof. by elim: $s=>/ /=x$ s $\rightarrow$. Qed.

Lemma count_map a $s:$ count a $(\operatorname{map} s)=$ count $($ preim $f$ a) $s$.

Proof. by elim: $\mathrm{s}=>/ /=\mathrm{x}$ s $\rightarrow$. Qed.

- $13 \%$ of clusters consist of theorems that are used in the proofs of other theorems of the same cluster.

Example 3.3. ML4PG discovers that the following two lemmas are in the same cluster:

Lemma altP : alt_spec b.

Lemma boolP : alt_spec b1 b1 b1. Proof. exact: (altP idP). Qed. 
- $11 \%$ of clusters are formed by "view" lemmas, an important kind of lemmas that are used in SSReflect to apply boolean reflection [16].

Example 3.4. ML4PG finds a cluster with the following two view lemmas coming from the fintype library:

Lemma unit_enumP : Finite.axiom [::tt]. Proof. by case. Qed.

Lemma bool_enumP : Finite.axiom [:: true; false].

Proof. by case. Qed.

- $5 \%$ of the clusters contain equivalence lemmas that are proven just by simplification.

Example 3.5. An example of this kind of clusters is given by the cluster that contains the following lemmas:

Lemma multE : mult $=$ muln. Proof. by []. Qed.

Lemma mulnE : muln = muln_rec. Proof. by []. Qed.

Lemma addnE : addn = addn_rec. Proof. by []. Qed.

Lemma plusE : plus = addn. Proof. by []. Qed.

- $4 \%$ of the clusters consist of lemmas that are solved using analogous lemmas.

Example 3.6. An example of clusters that consists of lemmas that are solved using analogous lemmas is the one containing the following two lemmas.

Lemma addnAC : right_commutative addn.

Proof. by move=> m $\mathrm{n} \mathrm{p}$; rewrite - ! addnA ( $\operatorname{addnC} \mathrm{n}$ ). Qed.

Lemma subnAC : right_commutative subn.

Proof. by move=> m n p; rewrite - !subnDA addnC. Qed.

Namely, the lemma subnDA (forall ( $a$ b c : nat), $a-(b+c)=(a-b)-c$ ) could be obtained from lemma addnA (forall ( $a b c$ : nat), $a+(b+c)=a+b+c$ ) using techniques like lemma analogy 20$]$.

In the case of heterogeneous clusters (clusters that include lemmas from different libraries), ML4PG discovers 154 clusters. In this case, the size of the clusters is bigger than in the case of homogeneous clusters; namely, the mean size is 8 lemmas per cluster. The different clusters can be classified as follows.

- $31 \%$ of the clusters contain lemmas that state properties applicable to several operators from different libraries.

Example 3.7. ML4PG discovers a cluster containing lemmas about the associativity of the addition of natural numbers (addn function) and the associativity of the concatenation of lists (++ operator).

Lemma catA s1 s2 s3: s1 ++ s2 ++ s3 = (s1 ++ s2) ++ s3.

Proof. by elim: $\mathrm{s} 1=>/ /=\mathrm{x}$ s1 $\rightarrow$. Qed.

Lemma addnA : associative addn.

Proof. by move=> m $\mathrm{n} \mathrm{p}$; rewrite (addnC $\mathrm{n}$ ) addnCA addnC. Qed.

- $27 \%$ of the clusters consist of lemmas related to operations over the base case of types.

Example 3.8. As an example of this kind of clusters, ML4PG discovers that there is a strong correlation among the following four lemmas: 
Lemma andTb : left_id true andb. Proof. by []. Qed.

Lemma orFb : left_id false orb. Proof. by []. Qed.

Lemma mulon : left_zero 0 muln. Proof. by []. Qed.

Lemma sub0n : left_zero 0 subn. Proof. by []. Qed.

- $12 \%$ of the clusters come from lemmas whose proof rely on the fundamental lemmas.

Example 3.9. ML4PG discovers a cluster with the following two lemmas about rot (that rotates a list 1 left $\mathrm{n}$ times) and the expn (exponentiation function).

Lemma $\operatorname{rot} 0 \mathrm{~s}: \operatorname{rot} 0 \mathrm{~s}=\mathrm{s}$.

Proof. by rewrite /rot drop0 take0 cats0. Qed.

Lemma expn_eq0 $\mathrm{m} \mathrm{e}:\left(\mathrm{m}^{\wedge} \mathrm{e}==0\right)=(\mathrm{m}==0)$ \&\& $(e>0)$.

Proof. by rewrite !eqnONgt expn_gt0 negb_or -lt0n. Qed.

At first sight, it seems that the only similarity between these two lemmas is that they only use rewriting rules in their proofs, however if we carefully inspect the lemmas that are used for rewriting, we notice that most of them are fundamental lemmas about nil (the base constructor for the list type) and 0 (the base constructor for the nat type).

- $9 \%$ of the clusters combine lemmas from the libraries about lists and natural numbers - note that the definition of lists and natural numbers is quite similar, both have one base case and a recursive one, so several lemmas are solved applying induction and using the inductive hypothesis.

Example 3.10. An example of this kind of clusters is given by the one that consists of the following lemmas:

Lemma catrev_catr $s t u$ : catrev $s(t++u)=$ catrev $s t++u$.

Proof. by elim: $s t \Rightarrow / /=x$ s IHs $t$; rewrite - IHs. Qed.

Lemma mulnDl : left_distributive muln addn.

Proof.

by move $=>\mathrm{m} 1 \mathrm{~m} 2 \mathrm{n}$; elim: $\mathrm{m} 1 \Rightarrow / /=\mathrm{m} 1 \mathrm{IHm}$;

rewrite -addnA -IHm.

Qed.

Lemma mem_cat $\mathrm{x}$ s1 s2:

$(\mathrm{x} \backslash$ in $\mathrm{s} 1++\mathrm{s} 2)=(\mathrm{x} \backslash$ in $\mathrm{s} 1) \|(\mathrm{x} \backslash$ in $\mathrm{s} 2)$.

Proof. by elim: $\mathrm{s} 1 \Rightarrow / /=\mathrm{y}$ s1 IHs; rewrite ! inE /= -orbA -IHs.

Qed.

In all these lemmas, we can see that induction is applied and after the use of some rewriting rules the inductive hypothesis is applied to finish the proof.

The similarity of most clusters ( $83 \%$ of them) can be easily discovered just inspecting the statement of the lemmas and their proofs. However, clustering is a statistical tool and there can be marginal cases, when one of the following situations arise:

$S 1$ The correlation of proof features is weak, e.g. it is a "leftover" cluster containing proofs that were not grouped with other strongly correlating clusters. In most of those cases, the clusters contain more than 10 elements, but it is difficult to find a common pattern followed by all lemmas.

$S 2$ There may be a strong feature correlation within a cluster, but the user does not understand what it is.

For $S 1-S 2$, we suggest to use automaton-shape proof-analysis of ML4PG; as we explain in the next sections. The basic idea is that the automaton shows whether there was a strong feature 
correlation, and if yes, - what it was; so that the user can identify in which of the situations he currently is.

S3 The user may see the correlated features in the ML4PG automaton, but nevertheless does not find this a useful suggestion. For case $S 3$, there is little ML4PG can do but help the user to understand that his case is exactly $S 3$ (in which case, there is a strong correlation of $S 2$, but it is irrelevant). One could try modifying various ML4PG parameters (like granularity, clustering algorithm, proof library), in a search for a better cluster. But ultimately, the user's "desired" proof may simply be different from any previous proof; so there may not be a better pattern in the library.

The above results show that ML4PG can be useful to detect patterns in early stages of a development. Namely, it can be used to find relations among functions and their lemmas, common strategies followed in a library and fundamental lemmas applied in several proofs. Besides, if a user knows a library (e.g. the library defining natural numbers), ML4PG can show similarities between lemmas about natural numbers and lists, facilitating the use of the new library based on the previous knowledge of the user.

\section{User Scenario 2. ML4PG for Detecting Irrelevant Libraries}

An (abstract) sequential game can be represented as a tree with pay-off functions in the leaves, dictating the win or loss of each player when the game finishes there. Each internal node is owned by a player and a play of a game is a path from the root to a leaf. A strategy is a game where each internal node has chosen a child. A Nash equilibrium is a strategy in which no agent can change one or more of his choices to obtain a better overall result for himself. A strategy is a subgame perfect equilibrium if it represents/have a Nash equilibrium of every subgame of the original game.

In this scenario, we use ML4PG to analyse two Coq libraries that formalise that all sequential games have Nash equilibria in binary games (games where each internal node has two choices) 35] and in the general case [34]. Note that unlike the other benchmarks presented throughout the paper, the files presented here are developed using plain Coq instead of SSReflect. ML4PG adapts to this change automatically.

It would be natural to assume that the proofs involved in verification of the two results will be very similar, and thus one could potentially hope for proof-pattern re-use. However, close inspection of these libraries can reveal that the actual proof strategies used in both libraries are different. Without ML4PG, such "negative" discovery would require user time and experience in comparing Coq proofs. We instead give it as a challenge to ML4PG that takes only a few seconds to analyse the libraries. ML4PG loads the Coq files developed in [34,35] and a library about topological sorting [33] used in 34]. These Coq files include 145 theorems, and we choose the K-means algorithm and the value 5 as the granularity parameter to obtain clusters using ML4PG. ML4PG finds 32 clusters using those parameters, and their mean size is three elements per cluster. The question is: how can the user interpret these results, when he sees those 32 sets of approximately three lemmas/theorems on the Proof General screen? One way to understand the clusters is to analyse their relative statistics and the structure of proofs contained in the clusters, as we explain below. Another option that ML4PG provides is visualising the common proof patterns, as e.g. shown in Figures $1,2,46$.

The automata produced by ML4PG should be read as follows. The automaton represents the proof-patches ( 5 proof-steps) of the lemmas that were clustered together. There are at most 5 states in the automaton and the $i$ th state is given by the list of $i$ th proof-steps of the proof-patches. The transitions between the states represent the tactics applied in the $i$ th proof-steps. If the tactics of several $i$ th proof-steps are the same or are related, the transitions are merged - and the automaton shows only one transition, cf. Figure 1. If, on the contrary, the tactics are different, there will be several different transitions shown between the states; annotated with the different tactics. Finally, if features of any particular proof step played a special role in the process of associating the proof 


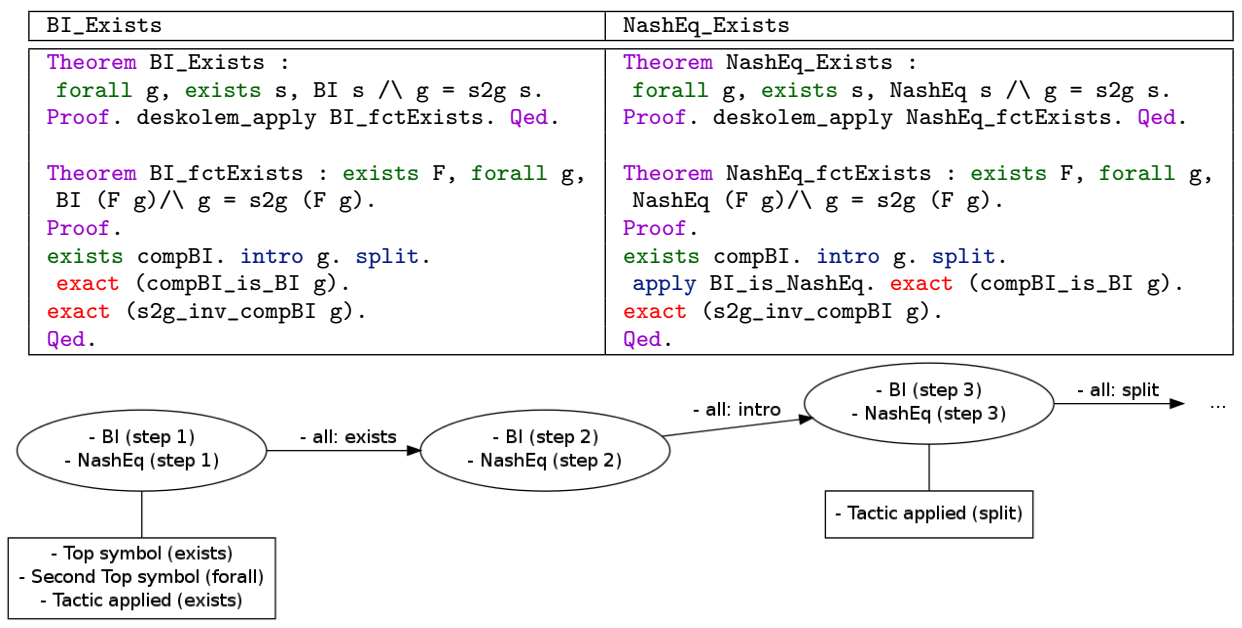

FIGURE 1. Top. Proofs of theorems BI_Exists and NashEq_Exists, coming from one library 35; and grouped together by ML4PG. Bottom. Fragment of the automaton-shape representation of the cluster containing the theorems. Note that there is always just one transition between the neighbouring states, which suggests that similar or same tactics are applied. According to the picture, the features with the highest correlation are the first two top symbols in the first goal; and the first and third applied tactics. Note that the second tactic - intro is not mentioned among the correlating features, as it is a common second-step tactic in many other proofs, outside of the cluster.

patches in the cluster, the correlated features annotate that proof-state and are shown in a square box (thus the box itself is not a state or part of the automaton).

It can be easily seen from ML4PG annotation of results that 21 of the 32 clusters $(65 \%)$ in the Nash libraries are homogeneous clusters, thus the similarity between the proofs within one library is higher than across libraries. Starting first with those homogeneous clusters, we notice that

- 8 clusters $(38 \%)$ contain lemmas about related functions, - as they use similar lemmas in their proofs.

Example 4.1 (Correlating homogeneous clusters in the Nash libraries - $S 2$ scenario). As an example of this kind of clusters, ML4PG discovers a cluster with two lemmas from [35]: the first one (BI_Exists) states that for every game, there exists a strategy that makes the game to have Backward-Induction equilibrium (each player plays optimally at every node); the second lemma (NashEq_Exists) states the analogous result for the Nash equilibrium. From the proofs of these two lemmas, it is clear that they use a similar sequence of tactics. This is also reflected in the automaton generated by ML4PG, see Figure 1. We know it is Scenario $S 2$, rather than $S 1$, as all transitions are single arrows, and the correlated features are shown by the automaton.

- 6 clusters $(28 \%)$ consist of lemmas about a concrete function.

Example 4.2. In [34, there is a function called StratPref that given an agent and two strategies decides which is the best one. ML4PG finds a cluster with two lemmas: the first one (StratPref_dec ) states the decidability of the function; and the second one states that the function produces an irreflexive relation.

- 4 clusters (19\%) contain theorems that use other theorems of the cluster in their proofs.

This quick analysis would show that some obvious grouping of proofs within one library was made by ML4PG. But, unless we are interested in a particular proof technique appearing in one of them, we direct our attention to patterns found across the libraries, hoping to find some common proof methods across the developments. 


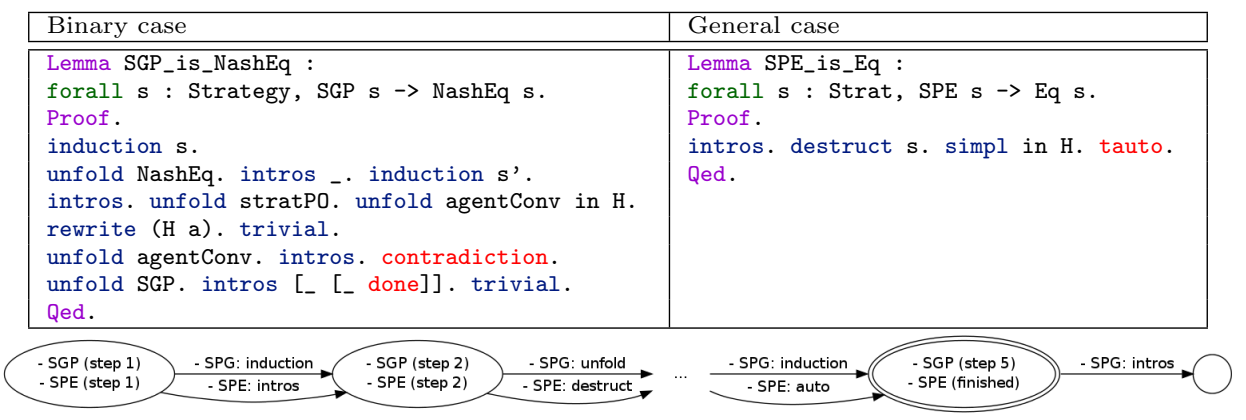

FIGURE 2. Top. Proofs of the theorems stating that Subgame Perfect Equilibrium implies Nash Equilibrium. The lemma statements are very similar; however, the structure of the proofs is completely different; hence, $M L 4 P G$ does not group these proofs together. Bottom. Fragment of automaton-shape representation of the proofs of the theorems. In contrast to the automaton of Figure 1 each proof step gives a separate transition (with a different tactic) and the proof-goals do not contain any features that correlate.

In the case of heterogeneous clusters, all the clusters consist of lemmas about auxiliary functions (for instance, about different properties of lists) that are common in all the libraries we are studying. However, there is no correlation among the important theorems of these libraries.

Example 4.3 (Disagreeing heterogeneous proofs in the Nash library). The proofs of the two main theorems of the two Nash libraries are given in Figure 2. Even if [34] is a generalisation of the work presented in [35], the proofs for Nash equilibrium are completely different, mainly for two reasons. First, the datastructures that are used in each development are too different, and therefore the lemmas about them do not have a strong correlation. In addition, the approaches that are used to prove them are completely different: one based on a procedure called backward induction [35] and the other is based on the fact that the preference of players is acyclic.

The above two proofs were not suggested in one cluster by ML4PG. However, if they were two "leftover" proofs, as Scenario $S 1$ explains, their automaton would look as Figure 2 suggests. The user would determine that it is an $S 1$ (rather than $S 2$ ) case, by noticing that transitions between every two neighbouring states are different, and no correlated features are shown by the automaton.

The results do not vary much when we try changing the clustering algorithm and the granularity values - reducing the granularity value produces bigger homogeneous clusters, but has little effect on heterogeneous clusters. As can be seen from this example, the ML4PG-based proof-pattern check could be an easy and fast way of getting the information about the absence of recyclable patterns across the libraries.

Note that in this case study, the total number of lemmas is smaller than in the previous casestudy (145 theorems); but the feature extraction mechanism of ML4PG automatically adapts to this, and handles statistics of small data sets as well as statistics of bigger data sets.

\section{User Scenario 3. A Team-Based Development}

In the last scenario, we turn to team-based applications of Coq and ML4PG. For this purpose, we translate the ACL2 proofs of correctness of the Java Virtual Machine (JVM) 23 into Coq/SSReflect. JVM [30] is a stack-based abstract machine which can execute Java bytecode. We have modelled an interpreter for JVM programs in Coq/SSReflect. From now on, we refer to our machine as "SJVM" (for SSReflect JVM).

An industrial scenario of interactive theorem proving may involve distribution of work-load across a team, and a bigger proportion of routine or repetitive cases. Here, the inefficiency often arises when programmers use different notation to accomplish very similar tasks, and thus a lot of work gets duplicated, see also [1]. We tested ML4PG in exactly such a scenario: we assumed that a 


\begin{tabular}{|c|c|c|c|}
\hline & & & \\
\hline & 0 & iconst 1 & \\
\hline & 1 & istore 1 & \\
\hline static int factorial(int $\mathrm{n}$ ) & 2 & iload 0 & \\
\hline\{ & 3 & ifeq 13 & Fixpoint helper_fact (n a) := \\
\hline int $a=1$. & 4 & iload 1 & match $\mathrm{n}$ with \\
\hline int $a=1$ & 5 & iload 0 & $10 \Rightarrow a$ \\
\hline while $(\mathrm{n} !=0)\{$ & 6 & imul & $\mathrm{S} p \Rightarrow$ helper fact $p(n * a)$ \\
\hline $\mathrm{a}=\mathrm{a} * \mathrm{n}$ & 7 & istore 1 & 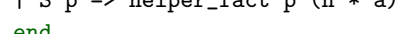 \\
\hline $\mathrm{n}=\mathrm{n}-1$ & 8 & iload 0 & \\
\hline$\}$ & 9 & iconst 1 & Definition $\mathrm{fn}$ fact $(\mathrm{n}: \mathrm{nat})$ \\
\hline return a; & $\begin{array}{l}10 \\
11\end{array}$ & $\begin{array}{l}\text { isub } \\
\text { istore } 0\end{array}$ & $\begin{array}{l}\text { Definition In_fact ( } \mathrm{n} \text { : nat) } \\
\text { helper_fact } \mathrm{n} 1 .\end{array}$ \\
\hline \} & 12 & goto 2 & \\
\hline & 13 & iload 1 & \\
\hline & 14 & ireturn & \\
\hline
\end{tabular}

FIGURE 3. Factorial function. Left: Java program for computing the factorial of natural numbers. Centre: Java bytecode associated with the Java program. Right: tail recursive version of the factorial function in Coq/SSReflect.

programming team is collectively developing proofs of the soundness of the specification, and the correctness of the implementation of Java bytecode for a dozen of programs computing multiplication, powers, exponentiation, and other functions about natural numbers. A new team member then tries to learn the important proof patterns while trying to prove similar results for a new function - factorial.

Given a specific Java method, we can translate it to Java bytecode using a tool such as javac of Sun Microsystems. Such a bytecode can be executed in SJVM provided a schedule (a list of thread identifiers indicating the order in which the threads are to be stepped), and the result will be the state of the JVM at the end of the schedule. Moreover, we can prove theorems about the SJVM model behaviour when interpreting that bytecode.

Example 5.1. The bytecode associated with the factorial program can be seen in Figure 3.

The state of the SJVM consists of 4 fields: a program counter (a natural number), a set of registers called locals (implemented as a list of natural numbers), an operand stack (a list of natural numbers), and the bytecode program of the method being evaluated.

Java bytecode, like the one presented in Figure 3, can be executed within SJVM. However, more interestingly than merely executing Java bytecode, we can prove the correctness of the implementation of the Java bytecode programs using Coq/SSReflect. For instance, in the case of the factorial program, the new team member is asked to prove the following theorem, which states the correctness of the factorial bytecode.

Theorem 5.2. Given a natural number $n$ and the factorial program with $n$ as an input, SJVM produces a state which contains $n$ ! on top of the stack running the bytecode associated with the program.

The proof of theorems like the one above always follows the same methodology adapted from ACL2 proofs about Java Virtual Machines [23] and which consists of the following three steps.

(1) Write in Coq/SSReflect the specification of the function and the algorithm, and prove that the algorithm satisfies the specification.

(2) Write the JVM program within Coq/SSReflect, define the function that schedules the program (this function will make SJVM run the program to completion as a function of the input to the program), and prove that the resulting code implements this algorithm.

(3) Prove total correctness of the Java bytecode.

Using this methodology, we have proven the correctness of several programs related to arithmetic (multiplication of natural numbers, exponentiation of natural numbers, and so on); see 21]. The proof of each theorem was done independently from others to model a distributed proof development.

Therefore, we simulate the following scenario. Suppose a new developer tackles for the first time the proof of Theorem 5.2 and he knows the general methodology to prove it and has access to the library of programs previously proven by other users. In this situation the different notations employed 


\begin{tabular}{|c|c|}
\hline Factorial & Exponentiation \\
\hline $\begin{array}{l}\text { Lemma fn_fact_is_theta } \mathrm{n}: \text { fn_fact } \mathrm{n}=\mathrm{n}^{\prime} ! \text {. } \\
\text { Proof. } \\
\text { rewrite /fn_fact. } \\
\text { by rewrite helper_fact_is_theta mul } 1 n . \\
\text { Qed. }\end{array}$ & $\begin{array}{l}\text { Lemma fn_expt_is_theta } n \mathrm{~m}: \mathrm{fn} \text { _expt } \mathrm{n} m=\mathrm{n} \text { ^m } \\
\text { Proof. } \\
\text { by rewrite /fn_expt helper_expt_is_theta } \\
\text { mul1n. } \\
\text { Qed. }\end{array}$ \\
\hline $\begin{array}{l}\text { Lemma helper_fact_is_theta } n a: \\
\text { helper_fact } n a=a * n ' ! . \\
\text { Proof. } \\
\text { move : } n \text { a; elim : } m=\text { [a m/ m IH } n \text { a /=]. } \\
\text { by rewrite /theta_fact facto muln1. } \\
\text { by rewrite IH /theta_fact factS } \\
\text { mulnA [a*_]mulnC. } \\
\text { Qed. }\end{array}$ & $\begin{array}{l}\text { Lemma helper_expt_is_theta } \mathrm{n} \mathrm{m} \text { a : } \\
\text { helper_expt } \mathrm{n} \mathrm{m} \mathrm{a}=\mathrm{a} *(\mathrm{n}-\mathrm{m}) \text {. } \\
\text { Proof. } \\
\text { move : a; elim : } \mathrm{n}=\text { [al } \mathrm{n} \text { IH a / }] \text {. } \\
\text { by rewrite /theta_expt expn0 muln1. } \\
\text { by rewrite IH /theta_expt expnS } \\
\text { mulnA [a * ] mulnC. } \\
\text { Qed. }\end{array}$ \\
\hline
\end{tabular}

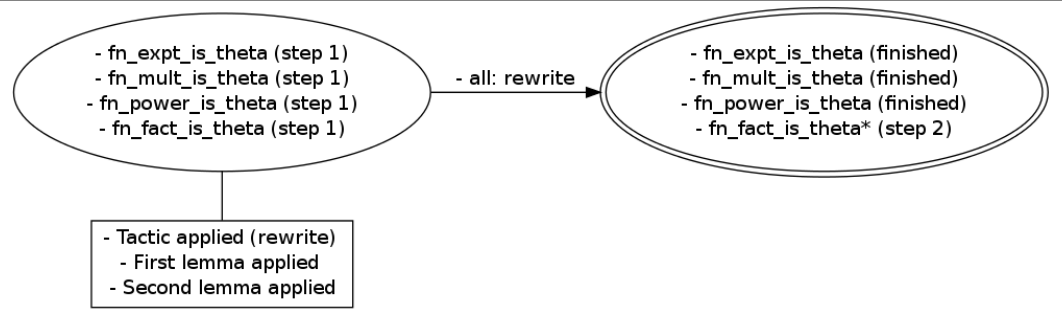

FIGURE 4. Top. Proofs of equivalence of the tail-recursive and recursive versions of functions exponentiation and factorial, following Proof Strategy 5.3 The left-hand-side shows a few initial proof steps for fn_fact_is_theta, leading to a deadlock. The right-hand-side shows the lemma (fn_expt_is_theta) suggested by $M L 4 P G$ (see Table 1) and an auxiliary lemma used to prove it. In italics is the proof reconstruction by analogy. Bottom. Automaton-shape representation of the proofs following Proof Strategy 5.3 The automaton only contains two states and its single transition is given by the rewrite tactic. Note that the three important features for this pattern are given by the applied tactic, the first lemma applied (a lemma that expands the definition of fn_fact and fn_expt) and the second lemma applied (the auxiliary lemma about the helper function). Note that the box explicitly suggests the lemmas to formulate for the proof of fn_fact_is_theta! (The * in the the second step of fn_fact_is_theta indicates the last proof step of the incomplete proof.)

by different users obscure some common features. ML4PG would be a good alternative to the manual search for proof patterns.

Let us focus on the first step of the methodology - that is, the proof of the equivalence between the specification of the factorial function (which is already defined in SSReflect using the function factorial having the notation $\mathrm{n}^{\prime}$ ! for factorial $\mathrm{n}$ ) and the algorithm, see Figure 4 . The Java factorial function is an iterative function; and the algorithm is written in Coq as a tail recursive function, see the right side of Figure 3. In the available SJVM libraries, all the tail recursive functions are defined using an auxiliary function, called the helper, and a wrapper for such a function. Discovering this fact is the first challenge for ML4PG. Suppose the new team member has stopped after one proofstep of trying to prove the lemma fn_fact_is_theta in a naive way, without a helper function; see Figure 4. He cannot proceed, and calls ML4PG for a hint. The suggestions provided by ML4PG in this case are the proofs of step (1) for three iterative programs: the multiplication, the exponentiation and the power of natural numbers. In addition, ML4PG produces the automaton-shape representation of Figure 4 showing the trace of the four proofs and the correlated features that determined the pattern. From the proofs of those lemmas, and the information in the automaton, it is easy to notice that all of them use an auxiliary lemma (like helper_expt_is_theta), and thus follow the same proof strategy:

Proof Strategy 5.3. Prove an auxiliary lemma about the helper considering the most general case. For example, if the helper function is defined with formal parameters $n, m$, and a, and the wrapper calls the helper initializing a at 0 , the helper theorem must be about (helper $n \mathrm{~m}$ a), not just about the special case (helper $n$ m 0 ). Subsequently, instantiate the lemma for the concrete case. 


\begin{tabular}{|c|c|c|c|c|c|}
\hline Algorithm: & $\begin{array}{c}g=1 \\
(n=16)\end{array}$ & $\begin{array}{c}g=2 \\
(n=18)\end{array}$ & $\begin{array}{c}g=3 \\
(n=21)\end{array}$ & $\begin{array}{c}g=4 \\
(n=24)\end{array}$ & $\begin{array}{c}g=5 \\
(n=29)\end{array}$ \\
\hline K-means & $30^{a, b, d}$ & $\mathbf{4}^{a-d}$ & $\mathbf{4}^{a-d}$ & $2^{c, d}$ & 0 \\
\hline E.M. & $21^{a-d}$ & $7^{a-d}$ & $7^{a-d}$ & 0 & 0 \\
\hline FarthestFirst & $28^{a-d}$ & $25^{a-d}$ & 0 & 0 & 0 \\
\hline
\end{tabular}

TABLE 1. A series of clustering experiments discovering Proof Strategy $5.3 \mid$ The table shows the sizes of clusters containing: a) Lemma about JVM multiplication program, b) Lemma about JVM power program, c) Lemma about JVM exponentiation program, and d) Lemma about JVM factorial program. The size of the data set is 147 lemmas, in bold (grey cells) is the cluster that finds exactly the four benchmark examples. Again, the lemmas grouped by clusters are consistently found for various algorithms and granularity values; and the K-means algorithm provides the most accurate clusters using 3 as granularity value.

The technical details are as follows. ML4PG correctly suggested similar lemmas to lemma fn_fact_is_theta. Table 1 shows the results for different choices of algorithms and parameters, and we highlight the most precise and helpful ML4PG result. In case the user is unsure of the optimal machine-learning parameters, he could use a "top-down approach". The highest granularity level does not produce any result. But, if we decrease the granularity level to 4, ML4PG spots some interesting similarities using the K-means algorithm. If this is not enough to discover Proof Strategy 5.3, one can decrease the granularity level to 3, for which ML4PG discovers four lemmas following the same general scheme.

On the basis of these suggestions, the new team member can try to reconstruct the missing auxiliary lemma and the missing proof steps in the main lemma by analogy. Figure 4 shows such analogical reconstruction in italics. This takes him through the first step of the general proof scheme.

In the second stage, he needs to prove that the Java bytecode implements the factorial algorithm. Again, after a few proof-steps, the user does not know how to continue the proof, see Figure 5 . If ML4PG is invoked at this point, it suggests three lemmas (using K-means algorithm and 3 as granularity value) that are used to prove that the three Java bytecode programs implement multiplication, exponentiation and power algorithms, respectively. All these Java bytecode programs are iterative and involve a loop, and it is easy to notice that the proofs follow the same proof strategy (see the automaton-shape representation in Figure 5):

Proof Strategy 5.4. Prove that the loop implements the helper using an auxiliary lemma. Such a lemma about the loop must consider the general case as in the case of Proof Strategy 5.3. Subsequently, instantiate the result to the concrete case.

Using this strategy and by analogy with the proofs of the other lemmas of the cluster, the user can finish the proof of lemma program_is_fn_fact; Figure 5 shows in italics the reconstruction of that proof.

Finally, it remains to prove the total correctness of the Java bytecode (Theorem 5.2). ML4PG finds that the proofs of the total correctness of 6 different programs are similar and follow the same proof pattern which consists of applying the lemmas obtained from steps (1) and (2), see Figure 6 . Again, Figure 6 illustrates the scenario of calling ML4PG on demand, and using its suggestions to reconstruct the proof by analogy. Following these guidelines, Theorem 5.2 can be formalised in Coq/SSReflect by analogy with a similar lemma for e.g. exponentiation, obtaining as a result the proof of the correctness of the factorial Java bytecode, as shown in Figure 6] see also 21 for the full proof.

The clusters found in the JVM scenario are heterogeneous since they belong to different libraries. The clusters obtained for the different steps consist of lemmas with the same proof structure and use analogous lemmas. This is an interesting kind of clusters since the analogous lemmas could be automatically generated using techniques presented in 20]. 


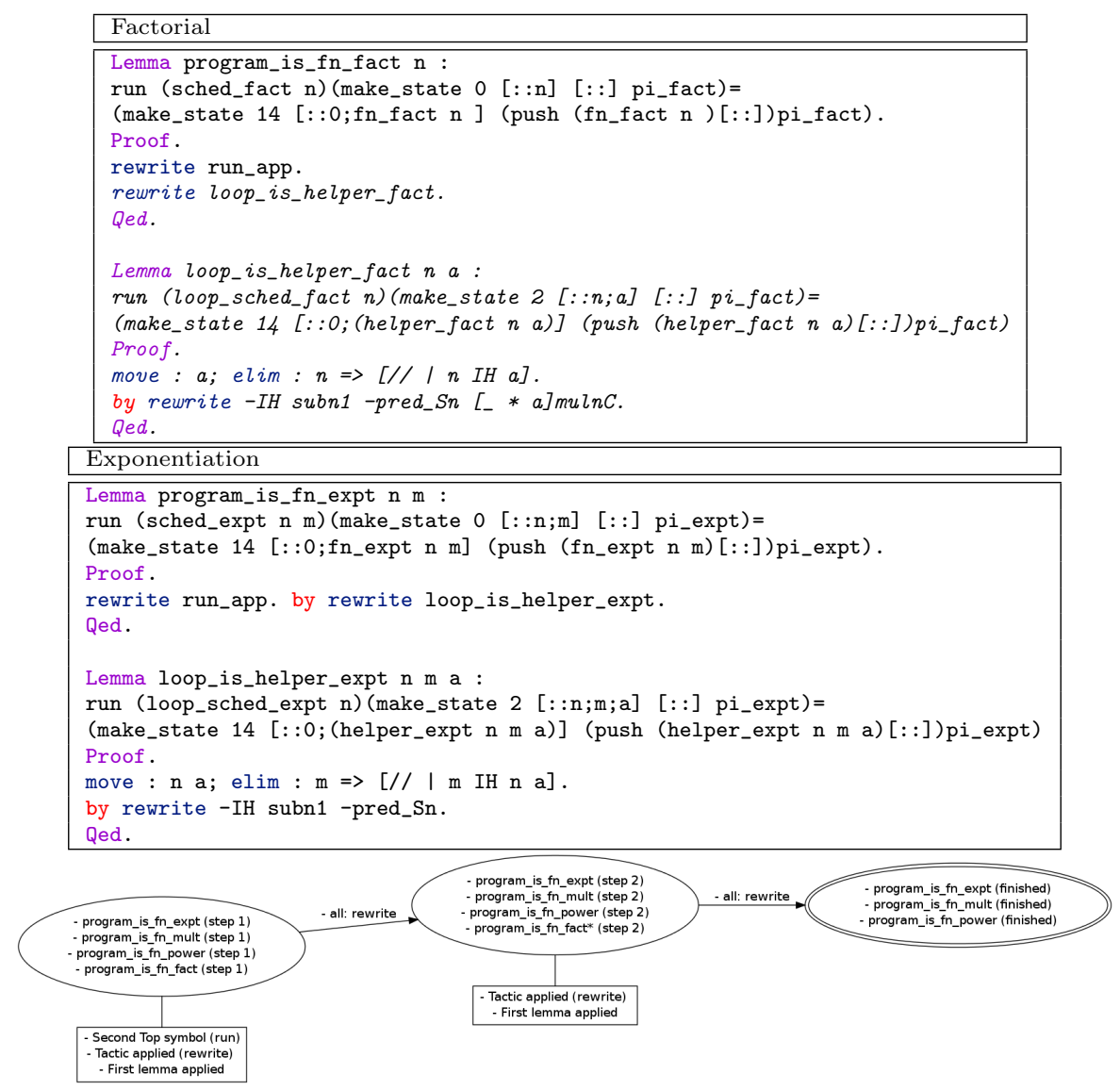

FiguRE 5. Two top tables. Proofs that the Java bytecode implements the factorial and exponentiation algorithms. When the user tries to prove program_is_fn_fact, he stops after one proof step (top table) and calls ML4PG. ML4PG suggests a few theorems, like program_is_fn_expt. It would work for e.g. K-means algorithm and granularity values from 1 to 4 , but using 4 as granularity value the cluster only contains these two lemmas. In italics, the user reconstructs the proof by analogy with program_is_fn_expt following Proof Strategy 5.4. Bottom. Automaton-shape representation of the four proofs following Proof Strategy 5.4 and discovered with granularity 3 . Note that all the proofs follow the same pattern: the application of the rewrite tactic two consecutive times. Additionally, two of the relevant features that determined the cluster were the lemma applied in the first rewriting tactic (lemma run_app); and the lemma applied in the second rewriting tactic (a lemma about the loop). Again, notice that ML4PG explicitly suggests which lemmas to analogise!

\section{ML4PG from the Domain Expert's Perspective}

During the formalisation of a theory, users of interactive theorem provers usually have the intuition that there is some kind of similarity or "proof-pattern" between their current development and other theories that they have previously created. The intuition of why two theorems are similar (or follow a common proof-pattern) varies from user to user, and is acquired with experience. Therefore, domain expert users with no experience using ITPs may have problems to find those similarities. We have seen in the previous scenarios that ML4PG can help to overcome this issue and can be used to guide domain expert users in the discovery of patterns in Coq proofs. We consider three kinds of domain expert users of ITPs: novice users of an unexplored domain in ITPs, novice users of a domain previously studied in ITPs, and advanced users; for all these users ML4PG can be helpful. 


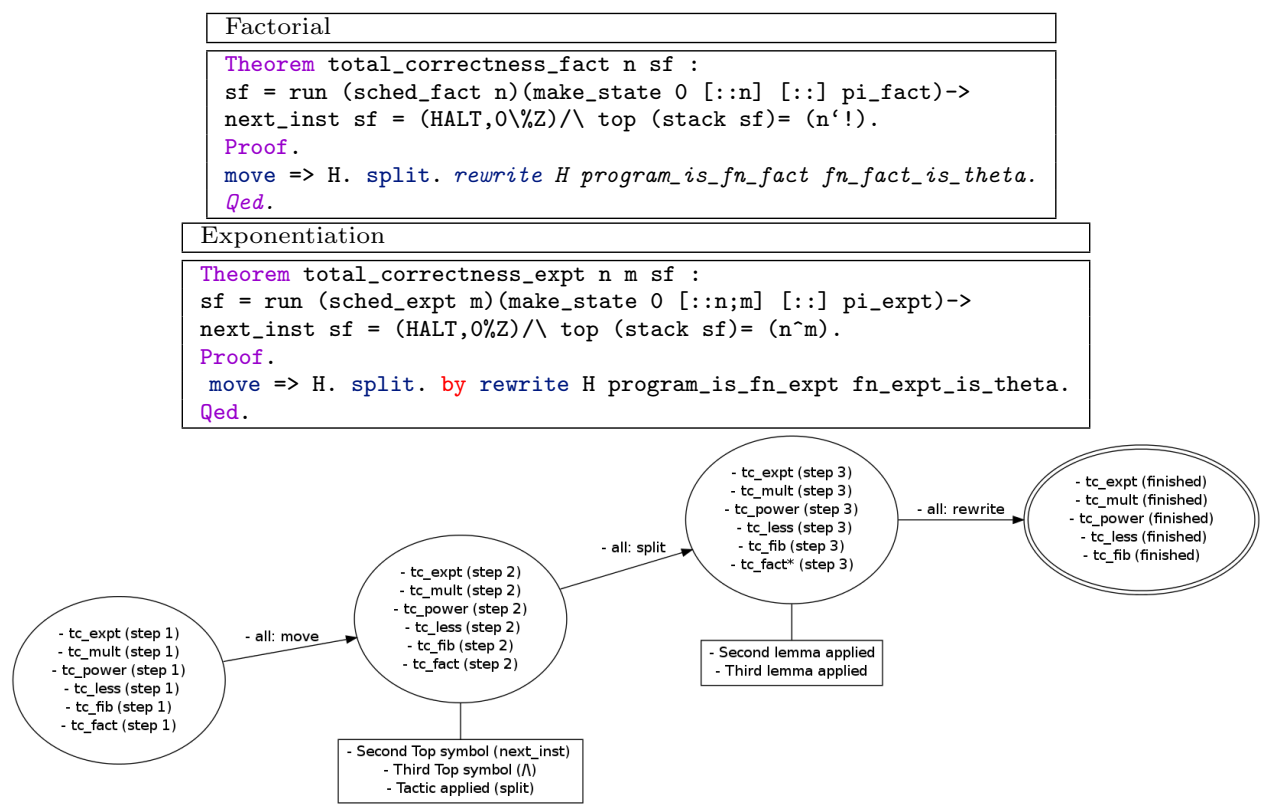

FIGURE 6. Top. Proofs of total correctness for exponentiation and factorial programs, cf. Theorem 5.2 The top table shows the initial step to prove Theorem 5.2 (total_correctness_fact). ML4PG suggests a few theorems, in particular, total_correctness_expt is suggested with e.g. K-means algorithm and any granularity values, but using 4 as granularity value gives the cluster containing only these two lemmas. In italics, the user reconstructs the proof by analogy with the theorem total_correctness_expt. Bottom. Automatonshape representation of the cluster of six theorems about the total correctness of JVM programs found with granularity value 3. Note again that there is always a single transition labelled by the same tactic, showing a strong correlation. In addition, the lemmas applied in the third proof step (lemmas the user would need to analogise) are two of the features that determined the pattern.

In the case of domains that have not been previously explored in ITPs, one of the reasons that was in the way for the adoption of ITPs was the lack of libraries with enough background material (for instance, if a domain expert needed a concrete result about sets for his development, he probably had to develop a whole library about set theory from scratch). This problem has been solved as a by-product of projects like the formalisation of the Feit Thompson Theorem [15] or the Flyspeck project [18, and nowadays there are big libraries containing several basic mathematical results. However, these libraries have grown so big that novice users can find difficulties to use them. Imagine that the user wants to explore the SSReflect library of User Scenario 1, inspecting the 1404 theorems one by one is unfeasible; on the contrary, ML4PG provides a snapshot of the library allowing the user to see groups of theorems that are similar. This provides the user a panoramic view of the results that are available and what are some of the common techniques used to prove them.

There are some domains where ITP experts have formalised some advanced notions [29] and domain experts are willing to extend those libraries with their own results. The advantage for novice users of these domains is that they can use the previous developments as guidance. This situation is similar to User Scenario 3; in this case, the users can use ML4PG not only as a discovery tool of the results that are already formalised, but also to obtain suggestions of similar results that were formalised in the same domain during the construction of a concrete proof.

Finally, domain experts that are advanced users of ITPs can also take advantage of ML4PG features. Even if an advanced user has the intuition that he is proving something similar to one of his previous developments, it can be difficult to remember where he did it. ML4PG can be used to 
explore the user's libraries and point out where are the proofs that follow a similar pattern to the current proof. This has a positive impact in the formalisation effort since, otherwise, the user should explore his libraries manually (probably re-executing the proofs) to find the theorems that are similar to his current proof-attempt.

Even if ML4PG can find patterns across different users, it works better finding patterns in the libraries developed by the same user. This is due to the fact that every user has his own style of proving and, therefore, the proof-patterns arise more clearly. This is also the reason why ML4PG works usually better with SSReflect than with plain Coq. SSReflect proofs use only a small number of tactics and this encourages a certain proof style; thanks to this, libraries developed by different people in big developments (e.g. the proof of the Feit Thompson Theorem) should always follow a concrete proof-style, facilitating the discovery of patterns by ML4PG.

\section{Conclusions and Future Work}

We have presented three scenarios, of very different nature and from different domains, to test the capabilities of statistical proof-pattern recognition. We have observed that ML4PG's feature extraction provides sufficiently robust results, tested using a few most common clustering algorithms (cf. Table 1). Judging by the experiments, the K-means algorithm is the most reliable algorithm, showing very stable results. The best value for granularity depends on the size of the library, in big libraries (cf. User Scenario 1) granularity values 4 and 5 return the most accurate clusters; however, in small libraries (cf. User Scenarios 2 and 3) the granularity value of 3 produces better results. ML4PG in general requires minimum user effort - mainly concerning adjustments of the granularity parameter to obtain the result of the required precision. ML4PG is very fast and gives instant outputs allowing the user to have quick search/evaluation in an interactive manner.

The most valuable feature of ML4PG is that it works equally well with any library we tried; irrespective of the subject domain or the size of the libraries. This property can be used to find patterns across subjects, libraries, and users; - as our case studies illustrate. Moreover, ML4PG discovers two kinds of clusters: homogeneous (all the lemmas of the cluster belong to the same library) and heterogeneous (the lemmas of the cluster belong to different libraries). Most of the time, the relation among the elements of a homogeneous cluster is clear (same proof structure, same lemmas or analogous lemmas). On the contrary, the relation among the elements of a heterogeneous cluster is more subtle (e.g. a general proof strategy or the use of some kind of auxiliary lemma).

Work is under way to incorporate the following extensions into ML4PG (see 22 for the most recent ML4PG versions):

- improve the conceptualisation and visualisation of proof-patterns; and,

- have a robust data-mining of type declarations and (co-)inductive definitions, alongside the currently used proof-analysis.

A longer-term project is to generate auxiliary lemmas and definitions automatically, on the basis of statistically discovered patterns. We have already done that for ACL2 [20]; however, extrapolation of the techniques of [20] from the first-order untyped language of ACL2 to the higher-order dependentlytyped language of Coq is a difficult task.

\section{Acknowledgments}

We would like to thank Marco Gaboardi and Vladimir Komendantsky for proof-reading the paper; their suggestions helped us to improve presentation.

\section{References}

[1] A. Amorim et al. A verified information-flow architecture. In 41st ACM SIGPLAN-SIGACT Symposium on Principles of Programming Languages (POPL'14), 2014. 
[2] A. Asperti et al. A Content Based Mathematical Search Engine: Whelp. In Post-Proceedings of the TYPES'04 International Conference, volume 3839 of LNCS, pages 17-32, 2006.

[3] A. Asperti et al. The Matita interactive Theorem prover. In 23rd International Conference on Automated Deduction (CADE'11), volume 6803 of LNCS, pages 64-69, 2011.

[4] D. Aspinall. Proof General: A Generic Tool for Proof Development. In 6th International Conference on Tools and Algorithms for the Construction and Analysis of Systems (TACAS'00), volume 1785 of LNCS, pages $38-43,2000$.

[5] D. Basin et al. Rippling: Meta-level Guidance for Mathematical Reasoning. Cambridge University Press, 2005.

[6] N. Benton. Machine Obstructed Proof: How many months can it take to verify 30 assembly instructions?, 2006.

[7] Y. Bertot and P. Castéran. Interactive Theorem Proving and Program Development, Coq'Art: the Calculus of Constructions. Springer-Verlag, 2004.

[8] C. Bishop. Pattern Recognition and Machine Learning. Springer, 2006.

[9] A. Blum. Learning boolean functions in an infinite attribute space. Machine Learning, 9(4):373-386, 1992.

[10] A. Bove et al. A Brief Overview of Agda - A Functional Language with Dependent Types. In 22nd International Conference on Theorem Proving in Higher Order Logics (TPHOLs'09), volume 5674 of LNCS, pages 73-78, 2009.

[11] A. Bundy et al. AI meets Formal Software Development (Dagstuhl Seminar 12271). Dagstuhl Reports, $2(7): 1-29,2012$.

[12] Coq development team. The Coq Proof Assistant Reference Manual, version 8.4. Technical report, 2012.

[13] H. Duncan. The use of Data-Mining for the Automatic Formation of Tactics. PhD thesis, University of Edinburgh, 2002.

[14] G. Gonthier. Formal proof - the four-color theorem. Notices of the American Mathematical Society, 55(11):1382-1393, 2008.

[15] G. Gonthier et al. A Machine-Checked Proof of the Odd Order Theorem. In 4th Conference on Interactive Theorem Proving (ITP'13), volume 7998 of LNCS, pages 163-179, 2013.

[16] G. Gonthier and A. Mahboubi. An Introduction to Small Scale Reflection. Journal of Formalized Reasoning, 3(2):95-152, 2010.

[17] A. Grabowski, A. Kornilowicz, and A. Naumowicz. Mizar in a nutshell. Journal of Formalized Reasoning, $3(2): 153-245,2010$.

[18] T. Hales. The Flyspeck Project fact sheet. Project description available at http://code.google.com/p/ flyspeck/, 2005.

[19] M. Hall et al. The WEKA Data Mining Software: An Update. SIGKDD Explorations, 11(1):10-18, 2009.

[20] J. Heras et al. Proof-Pattern Recognition and Lemma Discovery in ACL2. In 19th Logic for Programming Artificial Intelligence and Reasoning (LPAR-19), volume 8312 of LNCS, pages 389-406, 2013.

[21] J. Heras and E. Komendantskaya. ML4PG: downloadable programs, manual, examples, 2012-2014. http: //staff.computing.dundee.ac.uk/katya/ML4PG/

[22] J. Heras and E. Komendantskaya. Proof Pattern Search in Coq/SSReflect, 2014. http://arxiv.org/abs/ 1402.0081

[23] J S. Moore. Models, Algebras and Logic of Engineering Software, chapter Proving Theorems about Java and the JVM with ACL2, pages 227-290. IOS Press, 2004.

[24] M. Johansson et al. Conjecture synthesis for inductive theories. Journal of Automated Reasoning, 47(3):251-289, 2011.

[25] M. Kaufmann, P. Manolios, and J S. Moore, editors. Computer-Aided Reasoning: An approach. Kluwer Academic Publishers, 2000.

[26] E. Komendantskaya et al. Machine Learning for Proof General: interfacing interfaces. Electronic Proceedings in Theoretical Computer Science, 118:15-41, 2013.

[27] R. Krebbers and B. Spitters. Type classes for efficient exact real arithmetic in Coq. Logical Methods in Computer Science, 9(1):1-27, 2013. 
[28] D. Kühlwein et al. Overview and evaluation of premise selection techniques for large theory mathematics. In 6th International Joint Conference on Automated Reasoning (IJCAR'12), volume 7364 of LNCS, pages 378-392, 2012.

[29] C. Lange et al. A Qualitative Comparison of the Suitability of Four Theorem Provers for Basic Auction Theory. In Proceedings of Conferences on Intelligent Computer Mathematics (CICM'13), volume 7961 of LNCS, pages 200-215, 2012.

[30] T. Lindholm et al. The Java Virtual Machine Specification: Java SE 7 Edition, 2012.

[31] MATLAB. version 7.14.0 (R2012a). The MathWorks Inc., Natick, Massachusetts, 2012.

[32] T. Nipkow et al. Isabelle/HOL - A Proof Assistant for Higher-Order Logic, volume 2283 of LNCS. Springer, 2002.

[33] S. Le Roux. Acyclicity and finite linear extendability: a formal and constructive equivalence. In 22nd International Conference on Theorem Proving in Higher Order Logics (TPHOLs'09), Emerging Trends Proceedings, pages 154-169, 2007.

[34] S. Le Roux. Acyclic Preferences and Existence of Sequential Nash Equilibria: A Formal and Constructive Equivalence. In 20th International Conference on Theorem Proving in Higher Order Logics (TPHOLs'07), volume 5674 of LNCS, pages 293-309, 2009.

[35] R. Vestergaard. A constructive approach to sequential nash equilibria. Information Processing Letter, 97:46-51, 2006.

Jónathan Heras

School of Computing, University of Dundee, UK

e-mail: jonathanheras@computing.dundee.ac.uk

Ekaterina Komendantskaya

School of Computing, University of Dundee, UK

e-mail: katya@computing.dundee.ac.uk 